Iran Journal of Nursing (IJN)

Vol 32, No. 117, Apr 2019: 23- 32

\title{
Comparison of the Effects of Glycyrrhiza Glabra Vaginal Cream and Clotrimazole on the Symptoms of Fungal Vulvovaginitis
}

\author{
Neda Dodangeh ${ }^{1}$, Masoomeh Kheirkhah ${ }^{2}$, Jamileh Abolghasemi ${ }^{3}$, Faraz Mojab ${ }^{4}$ \\ Fatemeh Farshad ${ }^{5}$
}

\begin{abstract}
Background \& Aims: Vaginitis is a common cause of women's referrals to gynecology and obstetrics clinics as $75 \%$ of women have experienced the infection at least once, while approximately $45 \%$ have been affected twice or more. Candida albicans is responsible for $85-90 \%$ of fungal vaginal infections. Some of the predisposing factors in this regard are recent antibiotic use, pregnancy, diabetes, and use of contraceptives. The increased use of antibiotics has led to the higher prevalence of candida, and candida fungal vaginal infections are more prevalent and harder to eradicate during pregnancy. The growing interest of physicians and researchers in discovering new drugs has led to the increasing use of herbal products for the treatment of fungal infections with fewer side-effects in a more cost-effective manner compared to chemical drugs. Licorice belongs to the Fabaceae family and grows in southeastern Europe and southwestern Asia, including in Iran. The plant has anti-inflammatory and detoxifying properties and is used in the treatment of asthma, bronchitis, cough, peptic ulcer, and arthritis. The antiviral effects of herpes simplex type I have been confirmed in-vitro, as well as its synergistic effect with fluconazole on the treatment of fungal infections. Nevertheless, few clinical studies have been conducted in this regard, and further investigations have been recommended. The present study aimed to determine the effects of licorice on the treatment of fungal vaginal infections to suggest a drug with minimal side-effects and costs in the treatment of this common infection among women.

Materials \& Methods: This double-blind, three-blind, two-group randomized clinical trial was conducted on all the married women with fungal vulvovaginitis referring to Kamali Clinic affiliated to Alborz University of Medical Sciences, Iran. Eligible subjects were enrolled in the study in the case of positive fungal vaginal smear and stratified to two groups of intervention and control in a computerized manner. The inclusion criteria were women of the reproductive age (15-49 years), married women, no pregnancy, lactation or menopause, presenting with two symptoms of candida vaginitis (itching, burning and irritation, cheese-like discharge, burning urine, painful intercourse), absence of known chronic diseases (e.g., immunodeficiency, hypertension, diabetes), no therapies or use of herbal and chemical drugs for the treatment of genital infections within the past two weeks, absence of other vaginal infections (e.g., trichomoniasis, bacterial and mixed vaginitis), no use of creams and vaginal suppository 48 hours prior to the study, no participation in another research at the same time, no history of licorice allergies, no use of antibiotics and corticosteroids for the past two weeks for other medical reasons, and positive Sabouraud agar fungal test. The exclusion criteria were pregnancy during the treatment, allergies to licorice during the treatment, compulsive use of antibiotics for the treatment of systemic non-vaginitis infections during the treatment, and non-observance of the correct treatment method (forgetting to use the drug for more than one night). The sample size was determined by the McNemar test at 0.95 confidence level and 0.8 test power
\end{abstract}

\footnotetext{
1. MS in Midwifery, School of Nursing and Midwifery, Iran University of Medical Sciences, Tehran, Iran

2 . Assistant Professor, Department of Midwifery and Reproductive Health, Nursing Care Research Center, School of Nursing and Midwifery, Iran University of Medical Sciences, Tehran, Iran (Corresponding author)

Tel: 09124787691_ Email: kheirkhah.m@iums.ac.ir

3. Assistant Professor, Department of Biostatistics, School of Public Health, Iran University of Medical Sciences, Tehran, Iran

4. Professor, Department of Pharmacognosy, School of Pharmacy, Shahid Beheshti University of Medical Sciences, Tehran, Iran

5. Dentistry Student, Tehran University of Medical Sciences, Tehran, Iran
} 
considering $10 \%$ attrition, and 30 subjects were assigned to each group. Based on the sample size, the random allocation table with four blocks was prepared. After explaining the research objectives and obtaining informed consent from the subjects, they were stratified to the intervention and control groups using the table. Data were collected using an information registration form for the age, education level, occupation status, education level of the spouse, occupation status of the spouse, family income per month, housing status, history of menstruation and pregnancy, number of pregnancies, deliveries, menstrual patterns, methods of contraception, and disease and drug use history, history of drug allergies, and history of antibiotic use within the past two weeks. The patient's complaints were recorded in the symptom and clinical observation forms during the three visits. The evaluation checklist consisted of two sections for the recording of clinical observations and laboratory results. Clinical observations included vulvovaginal redness, cheese-like discharge, pustular papular lesions, vulvovaginal redness, and abnormal cervix, which were examined in the lithotomy position. With a sterile cotton swab that had been placed in an autoclave at the temperature of $120^{\circ} \mathrm{C}$ for 15 minutes before the examination, a sample was prepared from the secretions of the upper and lateral walls of the vagina, drawn on a slide, and sent to the laboratory. The laboratory results were recorded in a form based on the laboratory results. Licorice vaginal cream was prepared and packaged in the botanical laboratory of Shahid Beheshti University of Tehran (health code: 101301012003), and 1\% clotrimazole vaginal cream made by Pars Pharmaceutical Company was used as the basic treatment. In total, 60 completely identical vaginal creams with similar color, size, and shape coded A and B were provided to the researcher to be applied to the subjects based on the allocation table by an applicator inside the vagina for seven nights. The subjects were asked to refer to the center one week after the intervention with a coded card and a form for the recording of the signs and symptoms of the disease, which was completed daily by the samples. In addition, the patients were reminded of the time of the visits via phone, and the effects of the drugs on the signs and symptoms of the disease were examined to be recorded in the observation checklist. For the final confirmation of the presence or absence of the fungi, the sample smears were sent to the laboratory again. After data collection, data analysis was performed in SPSS version 16 using descriptive statistics (frequency, mean, and standard deviation) and inferential deviation (McNemar test and GEE).

Results: After the treatment, improvements were observed in the vaginal discharge, pruritus, irritation and burning, burning urine, painful intercourse, vulvovaginal redness, and cheese-like discharge. Furthermore, the McNemar test indicated a significant difference before and after the treatment in each group, while the GEE test showed no significant difference between the groups. Also, no significant differences were observed between the results of the laboratory examinations.

Conclusion: According to the results, licorice had therapeutic effects on the symptoms of fungal vaginal infection. Therefore, the use of licorice is suggested as an herbal medicine in the treatment of fungal vulvovaginitis.

Keywords: Vulvovaginitis, Fungal, Licorice, Clotrimazole

\section{Conflict of Interest: No}

How to Cite: Dodangeh N, Kheirkhah M, Abolghasemi J, Mojab F, Farshad F. Comparison of the Effects of Glycyrrhiza Glabra Vaginal Cream and Clotrimazole on the Symptoms of Fungal Vulvovaginitis. Iran Journal of Nursing. 2019; 32(117):23-32.

Received: 7 Jan 2019

Accepted: 10 Apr 2019 


\title{
مقايسه تأثير كرم وازينال شيرين بيان با كلوتريمازول بر علائم ولووازينيت قارجى
}

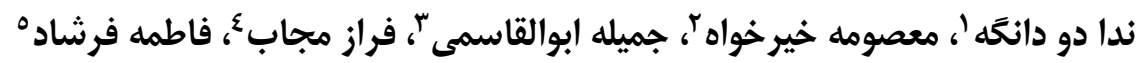

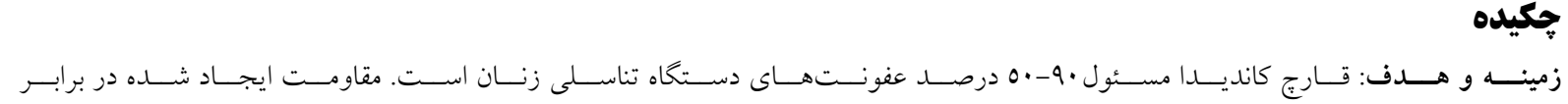

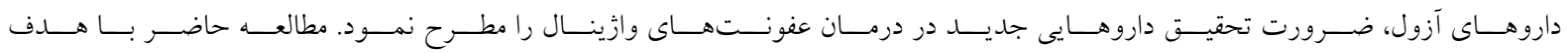

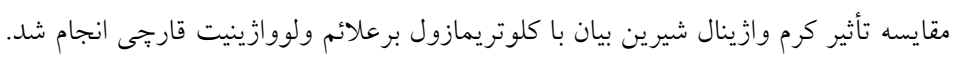

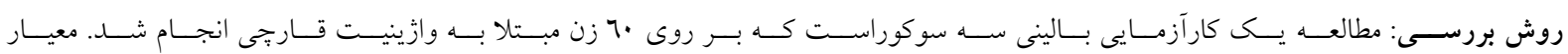

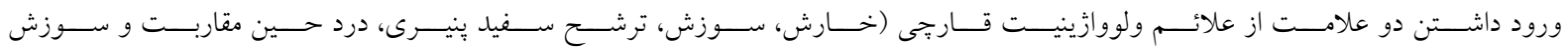

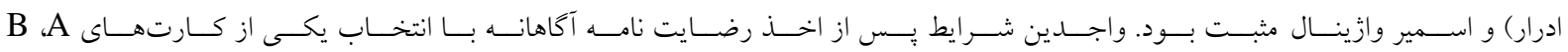

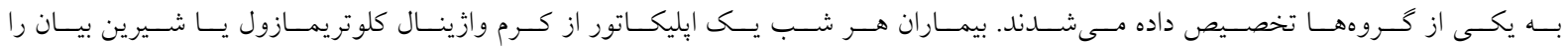

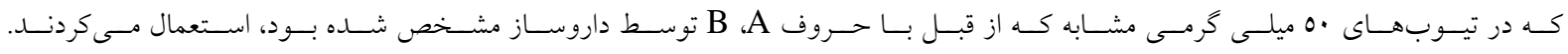

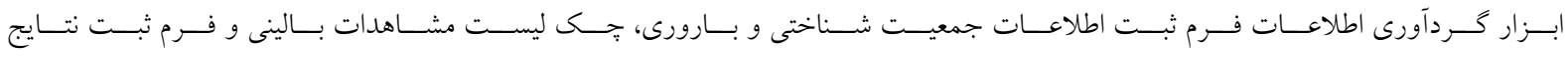

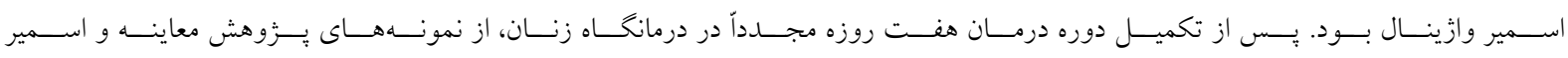

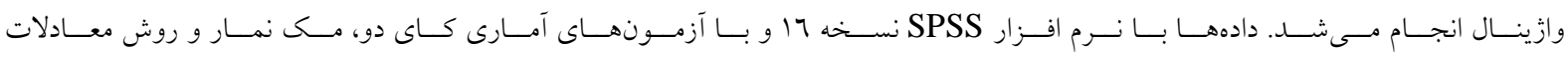

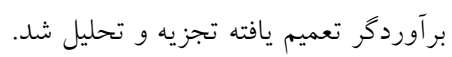

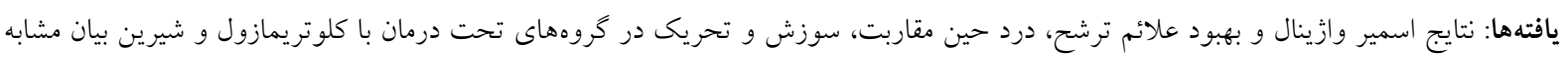

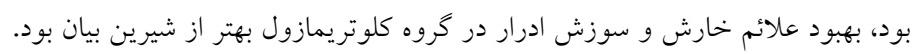

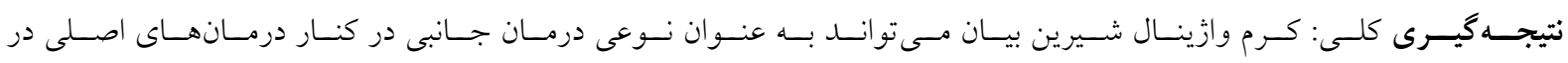

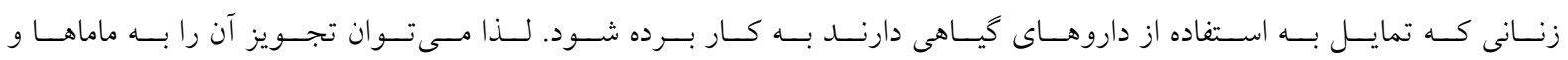
متخصصين زنان تو صيه نمود.

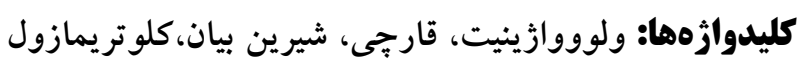
تعارض منافع: ندارد تاريخ دريافت: 9V/I•/IV تاريخ بذيرش:

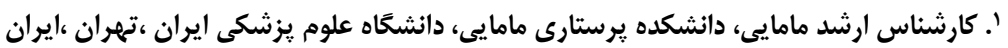

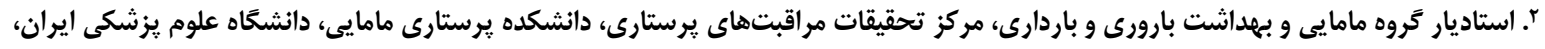
Email: kheirkhah.m@iums.ac.ir

شماره تماس: تهران، ايران. (نويسنده مسئول)

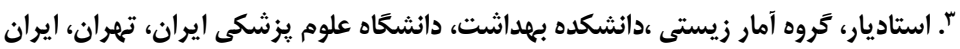

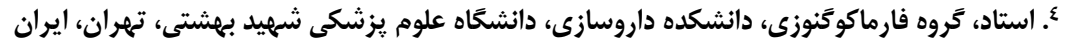

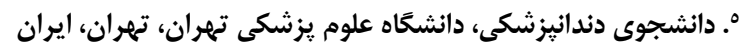


عصاره ريشه گياه شيرين بيان در برابر طيفى از ويروسها

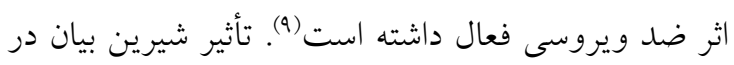
محيط آزمايشگاهى، بر كونههاى كانديداى وازينال تأييد شده و انجام مطالعه انسانى در اين زمينه توصيه كرديده

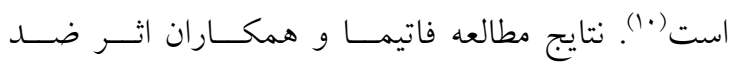
قـارجى اجزاى شيرين بيان از جمله كُابريدين بر قارج

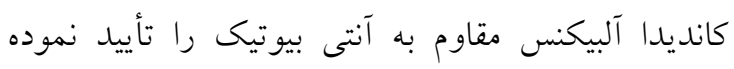
است (11) و اثر درمانى شيرين بيان در مبتلايان به كانديدياز

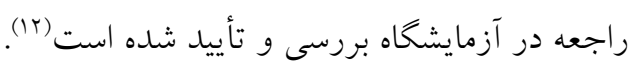

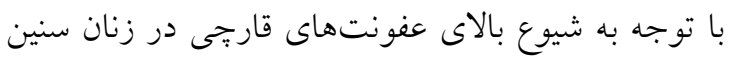

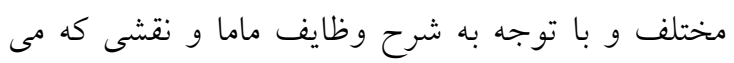
تواند در درمان بيمارىها با استفاده از داروهاى گياهى ايفا نمايد، انجام تحقيقات به منظور جمع آورى مستندات و شواهد علمى ضرورت دارد. لذا مطالعه حاضر با مقايسه تاثير كرم وازينال شيرين بيان و كلوتريمازول بر ولووازينيت قارجى طراحى و اجرا گردده است. تا بتوان طب مكمل ايرانى را با شواهد علمى به متخصصين زنان، ماماها و بانوان جامعه توصيه نمود.

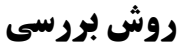
اين يزوهش يكى كار آزمايى بالينى سه سوكور با كروه كنترل

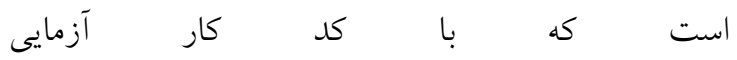
IRCT2017031133008N1

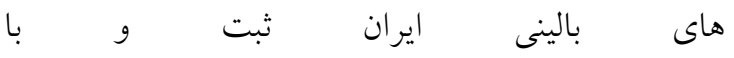
IR.IUMS.REC1395.9311373014 در كميته اخلاق دانشخاه علوم بزشكى ايران تأييد گرديده است. بـ بس

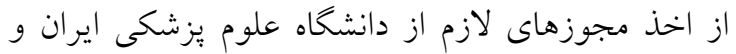

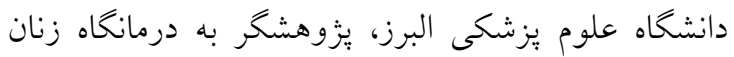
بيمارستان مراجعه نمود. با بيان اهداف يزوهش به بيمارني

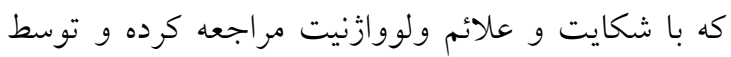
يزشك متخصص زنان مركز تشخيص بيمارى داده شده

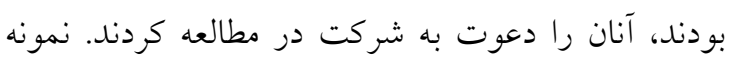

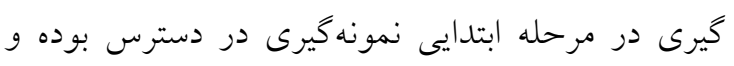

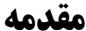

زنان در هملى گرووهاى سنى به عفونتهاى دستخاه

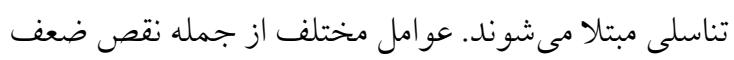

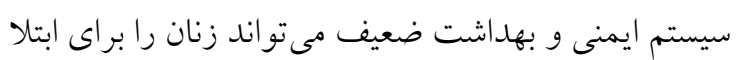

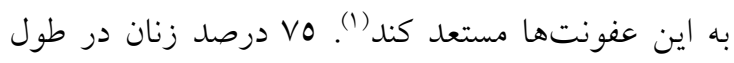

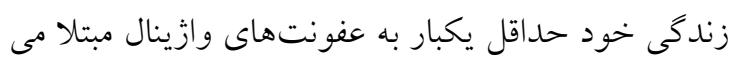

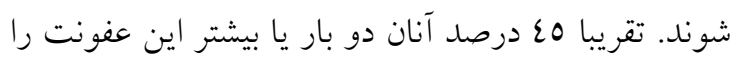
تجربه كرده و برخى در اثر عفونتهاى راجعه دجار مشكل

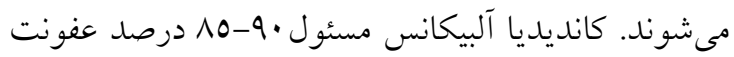
هاى قارجى وازن است و استفاده اخير از آنتى بيوتيكها،

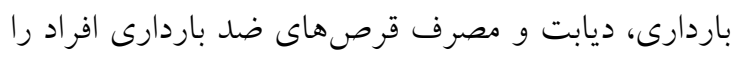

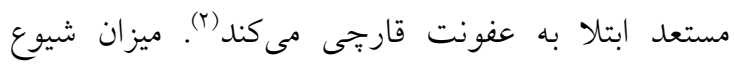

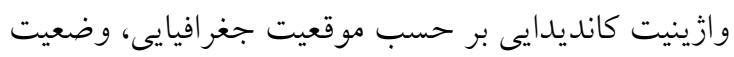

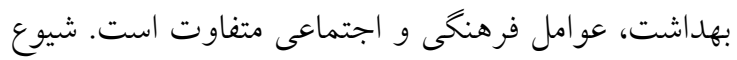

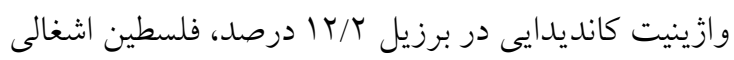

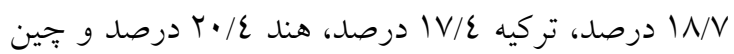

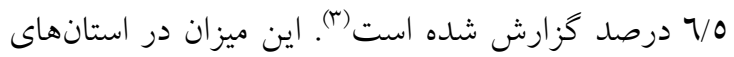
مختلف كشور ايران متفاوت است. بطورى كه در شهر مارد

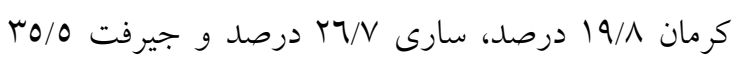
درصد گزارش شده است(ع). اخرجه اين بيمارى كشنده نيست ولى مى تواند به اختلال در روابط زناشويى منجر إنداك

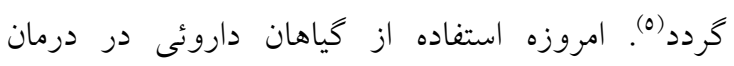
بيمارىها در مناطق مختلف رواج يافته است (1). كياه شيرين

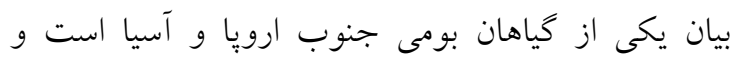

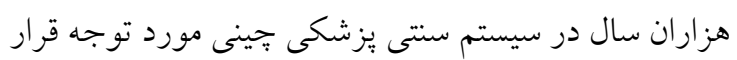

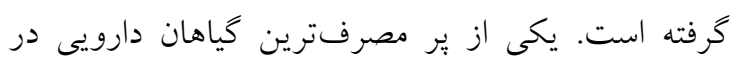

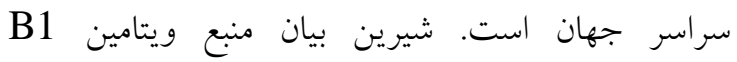
(تيامين)، B2 (نياسين)، B3 (ريبوفلاوين)، B5 (يانتوتنيك

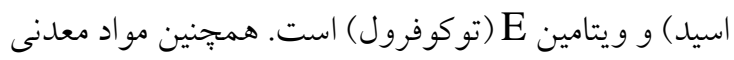
نظير فسفر، كلسيم، كلوئين، آهن، منيزيم، يتاسيم، سلنيوم، سيليسيم و روى را تأمين مىكند و غنى از بتا-كاروتن، كليسيريزين، كابريدين، ايزوليكويريتيخنين، تيمول، فنول،

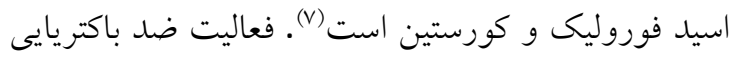

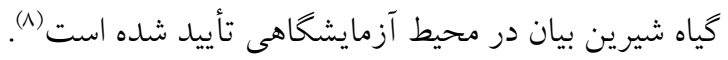


ينيرى، ضايعات بوسجولويايولار، قرمزى ولو و سرويكس

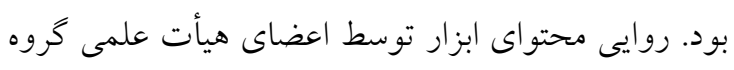

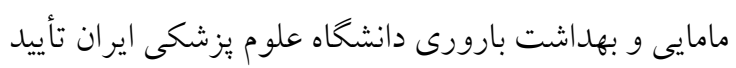

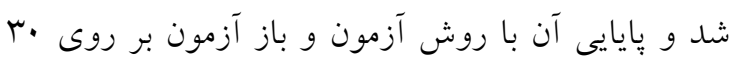

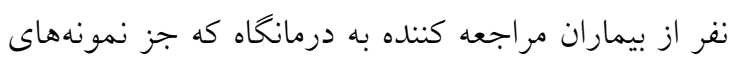

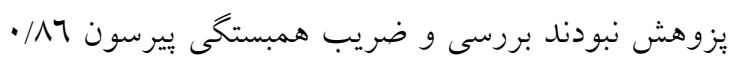

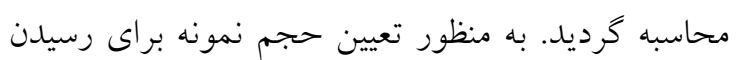

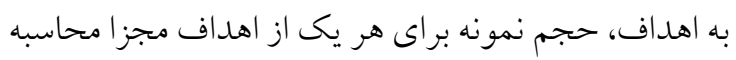

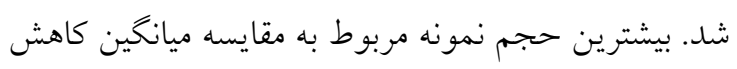

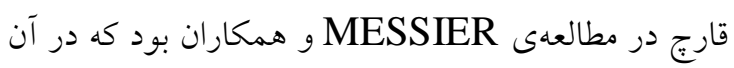

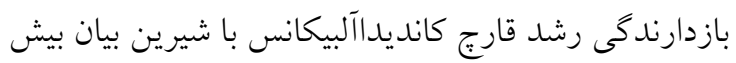

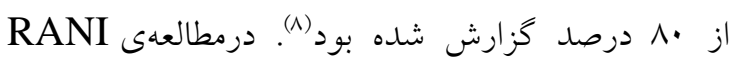

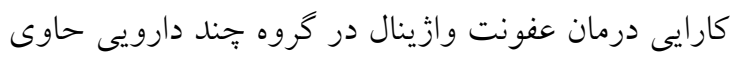

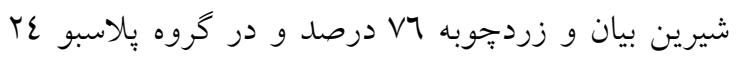

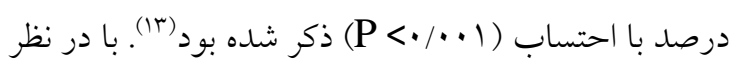

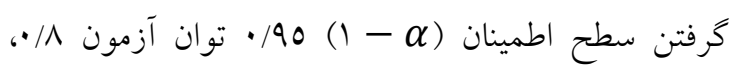

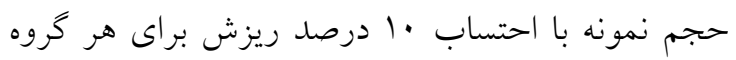

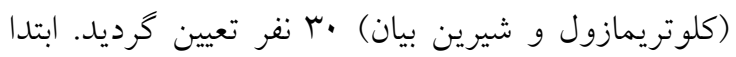
فرم مشخصات جمعيت شناختى با مصاحبه توسط

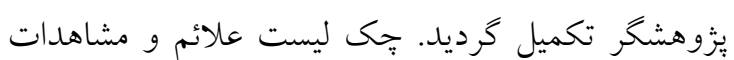

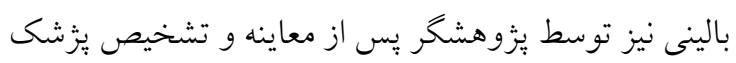

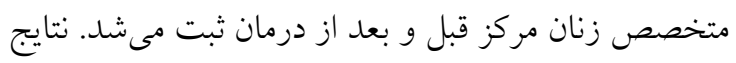

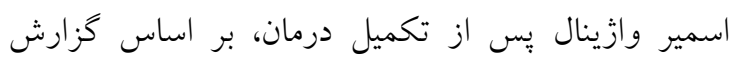
آزمايشگاه مركز در جهى ليست ثبت مى گرديد.

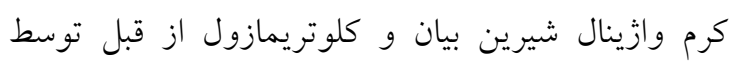
داروساز تيم تحقيق در تيوبهاى مشابه با كد A و B آماده

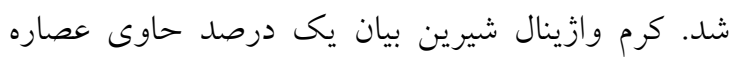

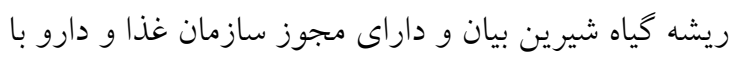

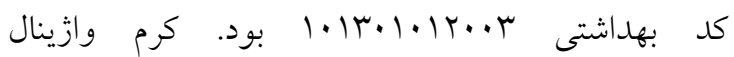

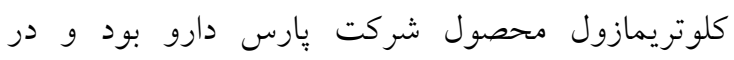

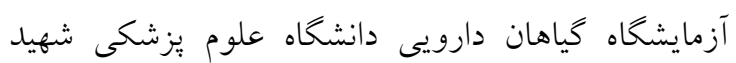

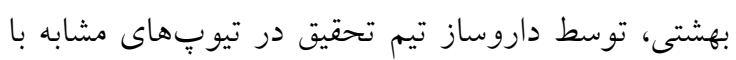
كد A و B مشخص و بسته بندى شده بود و تنها دكتر
معيارهاى ورود به مطالعه، داشتن سن 10 تا 9وع سال، داشتن

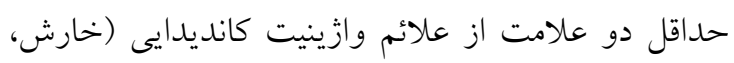
سوزش، سوزش ادرار، ترشحات ينيرى، درد حين مقاربت)

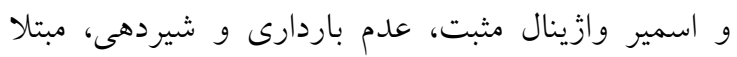

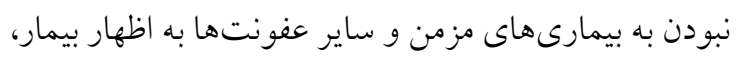

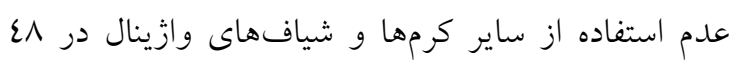

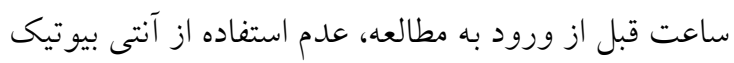
ها و داروهاى سركوبخر سيستم ايمنى طى دو هفته كذشته،

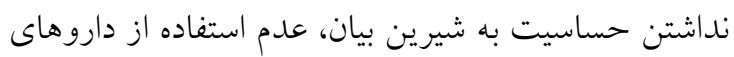

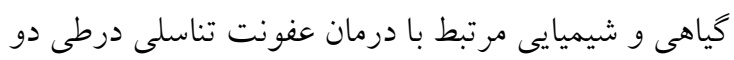

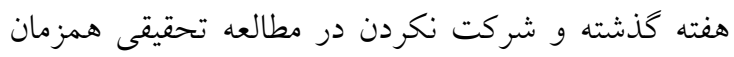

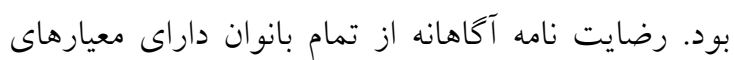

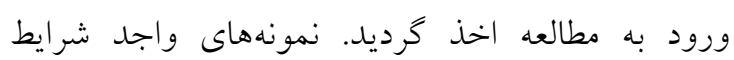

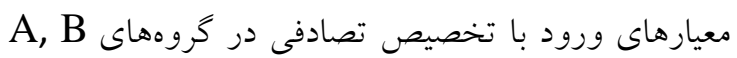
قرار كرفتند. به منظور تصادفى سازى ابتدا با استفاده از

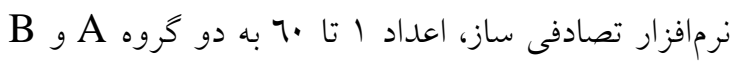

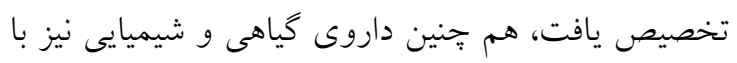

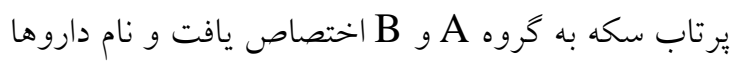
فقط با بر زّب A و B توسط دكتر داروساز مشخص

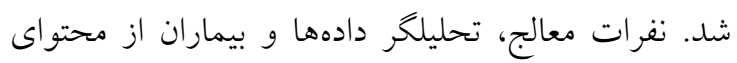

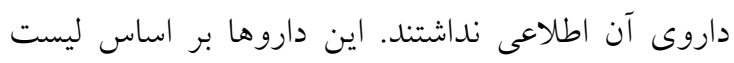

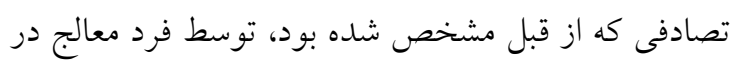

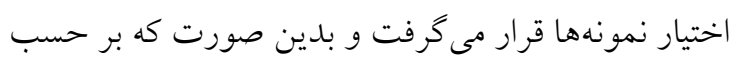

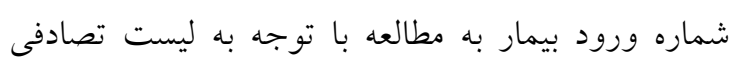

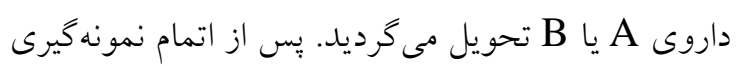

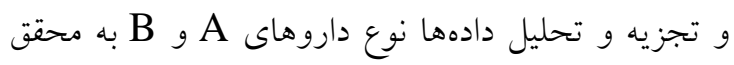

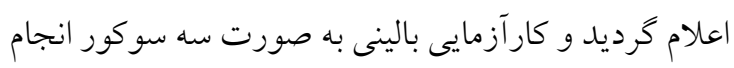
يذيرفت. ابزار گردآورى اطلاعات شامل فرم ثبت اطلاعات

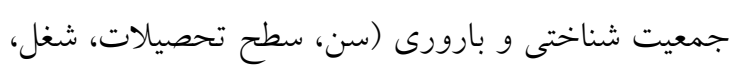
ميزان تحصيلات همسر، شغل همسر، سطح در آمد خانواده،

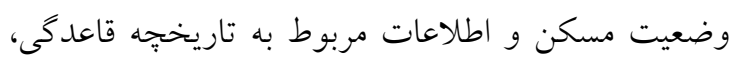

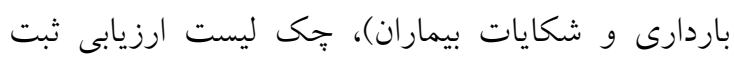

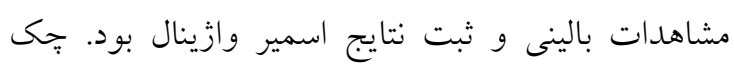

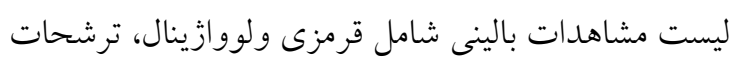


زمان مراجعه مجدد يادآورى مىشد. اثرات تجويز تيوب

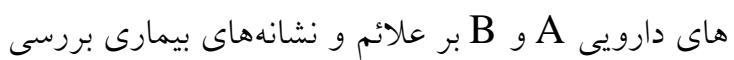

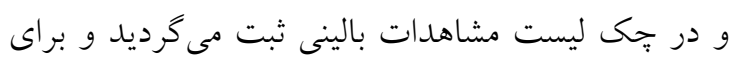

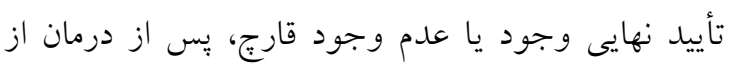

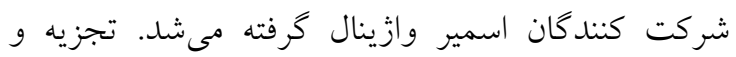
تحليل دادهها با استفاده از آزمونهاى مكى نمار، آناليز واريانس،كاى دو و روش معادلات برآورد كر تعميم يافته GEE(Generalized Estimation Equations)) استفاده از نرم افزار آمارى SPSS نسخه 77 ا، در سطح

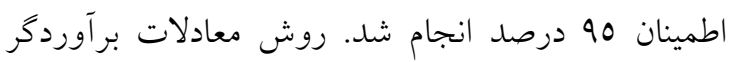
تعميم يافته روشى براى تسهيل و تحليل دادههاى

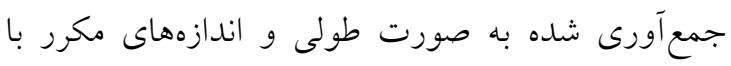
همبستخى نامشخص در مشاهدات مى باشد. در اين مطالعه

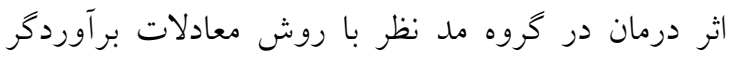

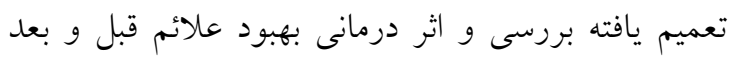
مصرف در شيرين بيان و كلوتريمازول قياس گرديد.

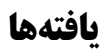

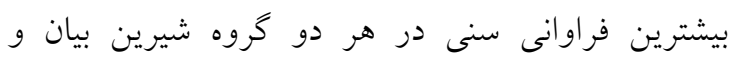

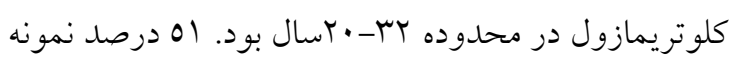

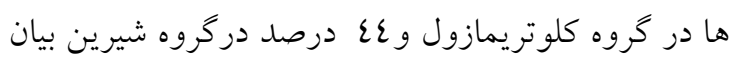

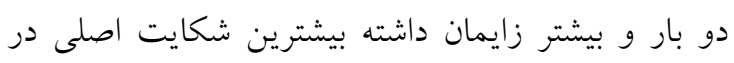

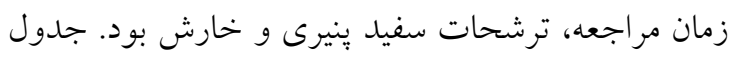

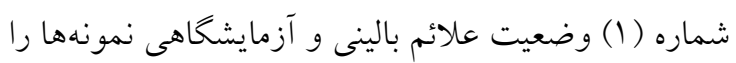
در هر دو گروه قبل و بعد از درمان نشان مىدهد.
داروساز از محتواى تيوبها اطلاع داشت، در اختيار يزوهشخر قرار گرفت. به منظور تصادفى سازى ابتدا با استفاده از نرم افزار تصادفى درفي

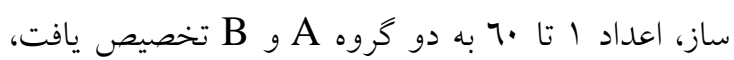

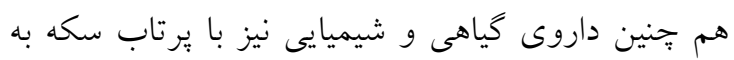
كروه A و B اختصاص يافت بر حسب شماره ورود بيمار

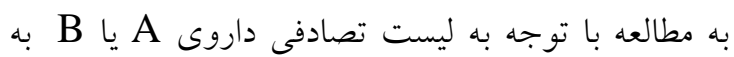

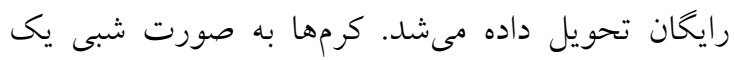
إيليكاتور به مدت هفت شب مورد استفاده قرار مى خرفت.

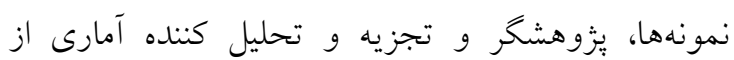

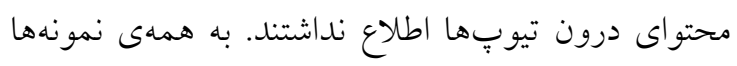

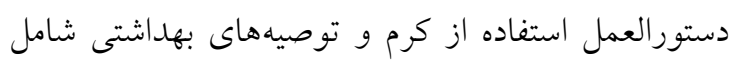
تعويض روزانه لباس زير، خشك كردن لباس زير با آفتاب

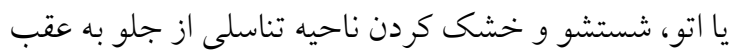

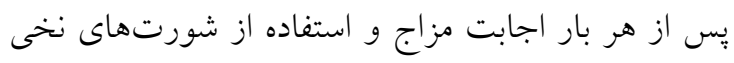
وكشاد توضيح داده مىشد. علاوه بر توضيح، بِمفلت بـانت

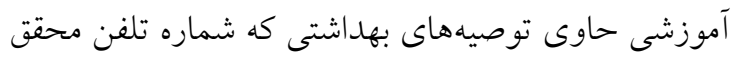
هم در آن ذكر شده بود، جهت مطالعه در منزل داده مى شعد. در صورت باردار شدن در حين درمان، حساسيت به شيرين

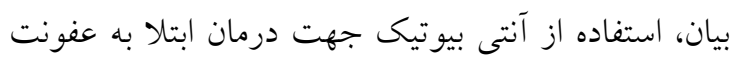

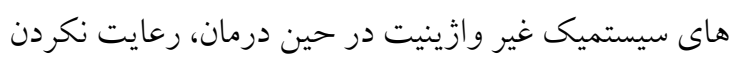

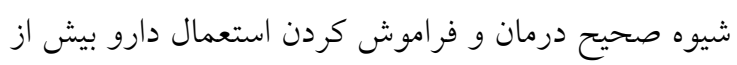

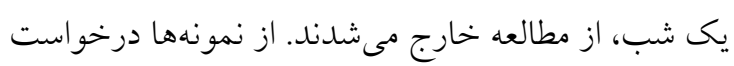

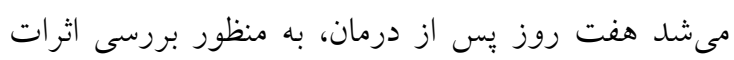

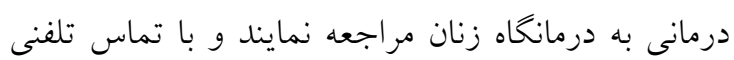




\begin{tabular}{|c|c|c|c|c|c|c|c|c|c|c|c|c|}
\hline \multirow{4}{*}{ نتيجه روش } & \multicolumn{5}{|c|}{ كلوتريمازول } & \multicolumn{5}{|c|}{ شيرين بيان } & \multicolumn{2}{|c|}{ علائم بالينى } \\
\hline & \multicolumn{3}{|c|}{ بعداز درمان } & \multicolumn{2}{|c|}{ قبل از درمان } & \multicolumn{3}{|c|}{ بعداز درمان } & \multicolumn{2}{|c|}{ قبل از درمان } & & \\
\hline & آزمون مك & درصد & تعداد & درصد & تعداد & آزمون & درصد & تعداد & درصد & تعداد & & \\
\hline & نمار & & & & & مكى نمار & & & & & & \\
\hline \multirow[t]{2}{*}{$\mathrm{P}=\cdot / \varepsilon \mid r$} & $r y \cdot r$ & $r$ & 1 & $9 \pi$ & TV & $r \mu / \varepsilon \varepsilon$ & 11 & $r$ & $9 \pi$ & TV & دارد & ترشح \\
\hline & $\mathrm{P}<\bullet / \cdots 1$ & QV & rA & V & r & $\mathrm{P}<\bullet / \cdots 1$ & 19 & $r 7$ & v & r & ندارد & \\
\hline \multirow[t]{2}{*}{$\mathrm{P}=\cdot / \cdot 1 \mathrm{~V}$} & $r y \cdot r$ & $r$ & 1 & VT & $r \varepsilon$ & $r \cdot / \cdot \varepsilon$ & IV & 0 & 19 & TV & دارد & خارش \\
\hline & $\mathrm{P}<\bullet / \cdots 1$ & QV & ru & 11 & 0 & $\mathrm{P}<\bullet / \cdots 1$ & N & ro & 11 & $r$ & ندارد & \\
\hline \multirow[t]{2}{*}{$\mathrm{P}=\cdot / \pi 79$} & $\mid r / \cdot V$ & . & . & $\varepsilon \varepsilon$ & 14 & $\varepsilon / 17$ & v & r & TV & $\wedge$ & دارد & درد حين \\
\hline & $\mathrm{P}<\bullet / \bullet l$ & $1 \cdots$ & rq & 00 & 17 & $\mathrm{P}<\bullet / \cdots 1$ & q & TV & $v r$ & rl & ندارد & مقاربت \\
\hline \multirow[t]{2}{*}{$\mathrm{P}=\cdot / 70$} & $19 / \cdot \varepsilon$ & $r$ & 1 & VT & YI & $1 N / \cdot 0$ & $1 \varepsilon$ & $\varepsilon$ & NT & $T \varepsilon$ & دارد & تحريك و \\
\hline & $\mathrm{P}<\bullet,, \cdot 1$ & $9 V$ & rs & rs & $\wedge$ & $\mathrm{P}<\bullet / \cdots 1$ & 17 & ro & 11 & 0 & ندارد & سوزش \\
\hline \multirow[t]{2}{*}{$\mathrm{P}=\cdot / \cdot \varepsilon_{0}$} & $19 / \cdot \varepsilon$ & . & . & VT & YI & $1 \% / \cdot 7$ & $1 \varepsilon$ & $\varepsilon$ & $\pi$ & 11 & دارد & سوزش \\
\hline & $\mathrm{P}<\bullet / \cdots 1$ & $1 \cdots$ & rq & ru & $\wedge$ & $\mathrm{P}<\bullet / \cdots 1$ & $\wedge 7$ & ro & r & 11 & ندارد & ادرار \\
\hline
\end{tabular}

خارش، بهبود تحريك و سوزش و · ا درصد در درمان درد حين مقاربت و سوزش ادرار بيماران مبتلا مؤثر بوده است. (جدول شماره ب).
شيرين بيان در بهبود ترشحات وازينال 19 درصد، خارش א در صد، سو درصد درد حين مقاربت، 17 درصد در بهبود تحريك و سوزش و 17 درصد در بهبود سوزش ادرار مؤثر بود. كلوتريمازول 9V درصد در بهبود ترشحات وازينال،

جدول شماره ז: مقايسه نتايج اسمير وازينال يس از درمان در گروههاى شيرين بيان و كلوتريمازول

\begin{tabular}{|c|c|c|c|c|c|c|}
\hline \multirow{5}{*}{$\begin{array}{c}\mathrm{df}=1 \\
\mathrm{P}=\cdot / \cdot \vee \wedge \\
X^{2}=r / l \cdot v\end{array}$} & \multicolumn{2}{|c|}{ كلوتريمازول } & \multicolumn{2}{|c|}{ شيرين بيان } & \multicolumn{2}{|c|}{ گروهها } \\
\hline & درصد & فراوانى & درصد & فراوانى & & \\
\hline & $r \cdot$ & 7 & rv & 11 & مثبت & نتايج اسمير \\
\hline & $\wedge \cdot$ & $T \varepsilon$ & r & 19 & منفى & وازينال \\
\hline & $1 \ldots$ & $r$. & $1 \cdots$ & $r$. & جمع & \\
\hline
\end{tabular}

از مشكلات طب جديد با وجود امتيازهاى ظاهرى آن نسبت به طب سنتى مصرف روزافزون داروهاى شيميايى است كه باعث مقاومت دارويى و عوارض جانبى مىشود. بر اين اساس پِ از مطالعه روى اثرات كياهان بشر اقدام

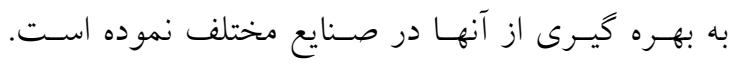

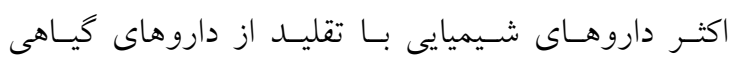

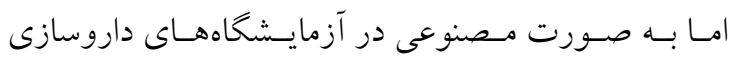
تهيه مىشوند. برآورد شده كه دست كم يـك سـوم از كليه فر آوردههاى مورد مصرف، منـشاء گيـاهى دارنــد (عاء).
بهبود علائم بالينى ترشح، درد حين مقاربت، سوزش و. تحريك و نتايج اسمير وازينال در گروههاى تحت درمان با تهان كلوتريمازول و شيرين بيان از نظر آمارى تفاوت معنىدار نداشت و اثرات مشابه است. علائم خارش و سوزش ادرار در كروه كلوتريمازول بهتر از شيرين بيان بهبود يافته است. 
نداد، اما عصارههاى اتانوليى و متانوليك شيرين بيان فعاليت قابل ملاحظهاى عليه گونههاى ايزوله شدهى

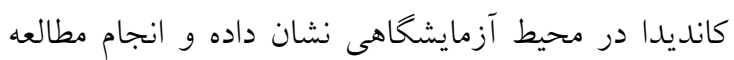

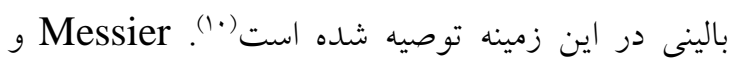
همكاران نيز در ايتاليا طى مرورى نظاممند تأثير عصاره توصيه

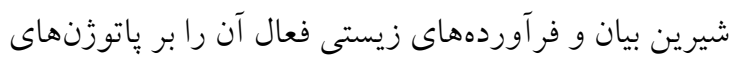

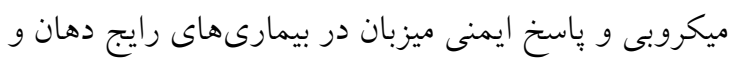

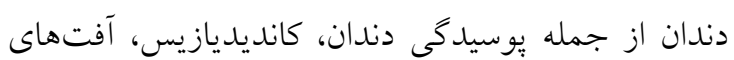

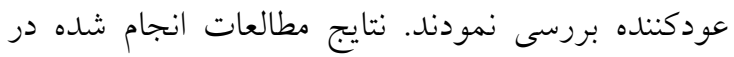

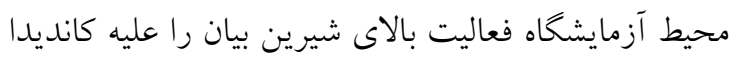

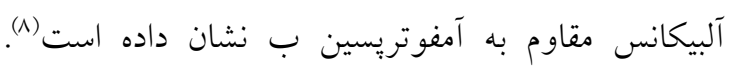

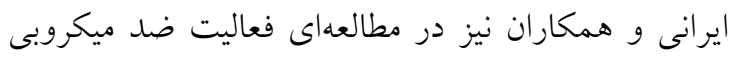

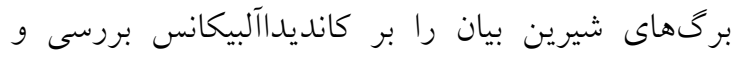

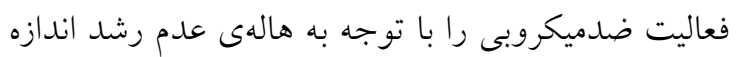

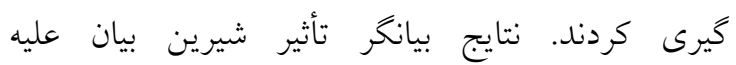
كانديدآلبيكانس بوده است (17). نتايج مطالعه SIRILUN و همكاران نيز نشان داد كه تجويز شيرين بيان مىتواند

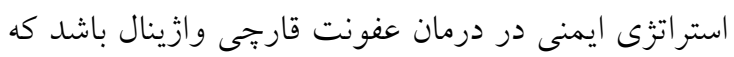

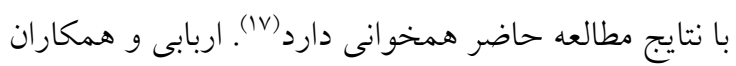
نيز به مقايسه اثرات ضدقارجى كياه شيرين بيان با نيستاتين در محيط آزمايشگاه يرداخته، نشان دادهاند كه هاله عدام

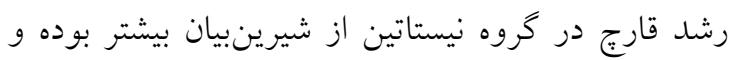

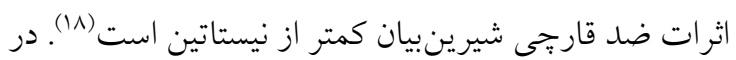

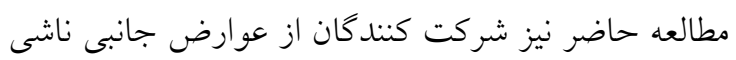

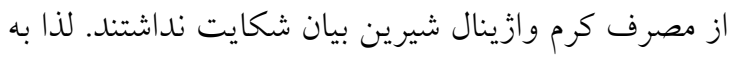
نظر مىرسد مىتوان اين فراورده گياهى را همراه با ساير داروهاى رايج در درمان عفونتهاى وازينال توصيه نمود.

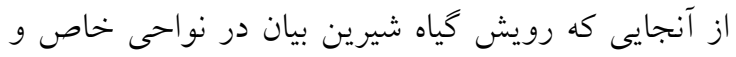
دسترسى بـه ريسشه و ريزوم و تهيه عصاره در فصول خاصى امكان بذير است، اين مسئله مىتواند از

محدوديتهاى استفاده از اين كياه دارويى باشد (عاصن) با توجه به كمبود مطالعه انسانى در مورد كياه شيرين بيان،

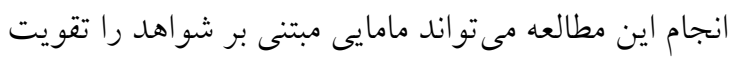
نموده و زمينه ساز ساخت و بهره بردارى بيشتر از كياهان
كَاه شيرين بيان كه از تيره لكومينوره و جز دسته سايونين كليكوزيدها است .مواد مؤثره در عصاره شيرين بين بيان

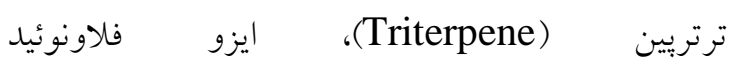
و كومارين (Isoflavonoids) طريق تأثير بر غشاى سيتويلاسمى و تغييرات مورفولوزيك

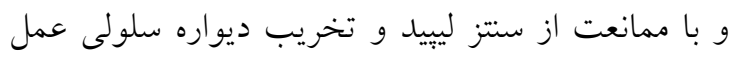

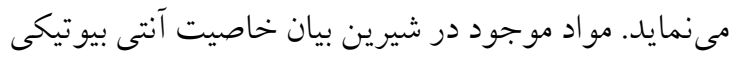
داشته و مىتوان از آن به عنوان آنتى بيوتيكى استفاده كرد.

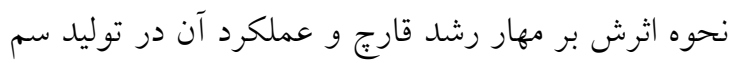

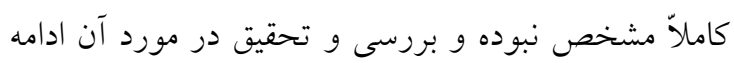

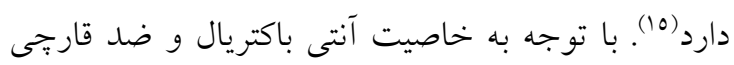
عصاره شيرين بيان مطالعه حاضر با هدف مقايسه تأثير كرم

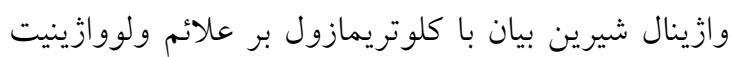

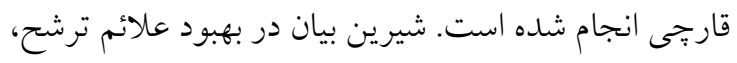

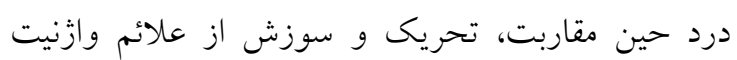

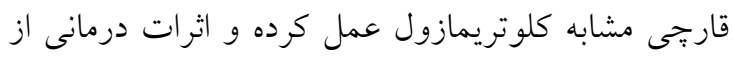
نظر آمارى تفاوت نداشت. اما تأثير درمانى در بهبود علائم خارش و سوزش ادرار در مصرف كنندكان كرم وازينال كلوتريمازول بهتر بود. از آنجايى كه استفاده از كياهان

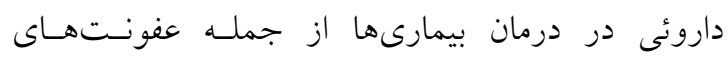

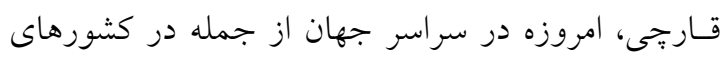

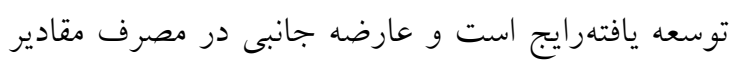
كم شيرين بيان كزارش نشده (7).

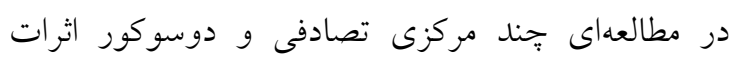

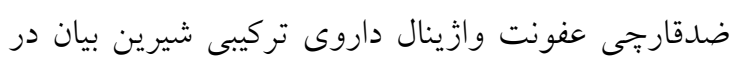
مقايسه با دارونما تأييد شده است (rا)، كه با نتايج مطالعه

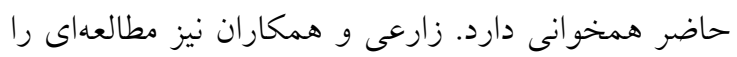

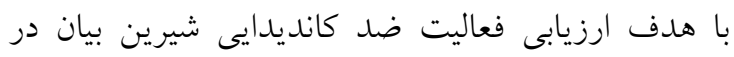

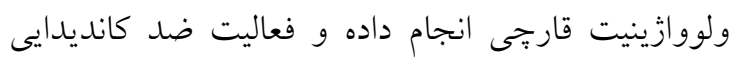
شيرين بيان اتانوليى و متانوليك را در برابر كانديداهاى

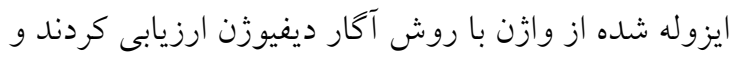

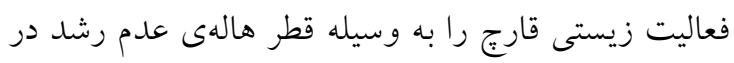

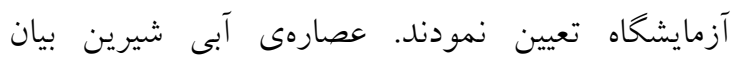

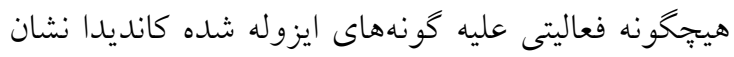




\section{تعارض منافع: هيج گ گونه تعارض منافع از سوى نويسندكان بيان نشده است.}

$$
\begin{aligned}
& \text { تقدير و تشكر } \\
& \text { اين مقاله حاصل بايان نامه كارشناسى ارشد مامايى نويسنده } \\
& \text { اول مىباشد. از زحمات معاونت يزووهشى و تحصيلات }
\end{aligned}
$$

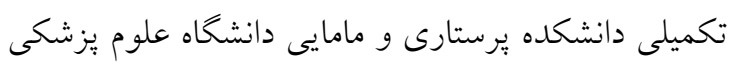

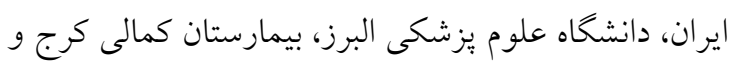

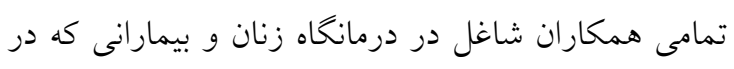

$$
\begin{aligned}
& \text { اين مطالعه شركت نمودند، قدردانى مىنماييم. }
\end{aligned}
$$

$$
\begin{aligned}
& \text { دارويى در درمان عفونتهاى وازينال باشد. در مطالعه اخير }
\end{aligned}
$$

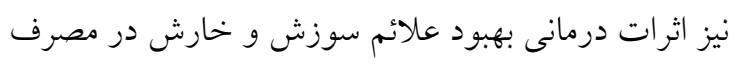

$$
\begin{aligned}
& \text { كنندكان كرم وازينال شيرين بيان كمتر از كرم وازينال } \\
& \text { كلوتريمازول بود كه نشان دهنده اين است كه بهتر است }
\end{aligned}
$$

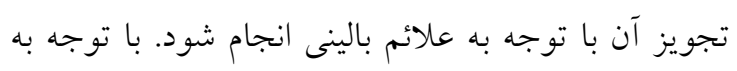

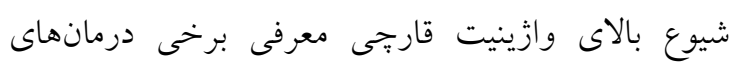

$$
\begin{aligned}
& \text { كياهى، با توجه به تاريخجهى كهن استفاده از اين داروها }
\end{aligned}
$$

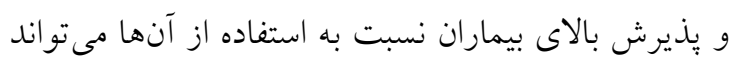

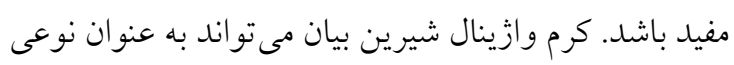

$$
\begin{aligned}
& \text { درمان جانبى در كنار درمانهاى اصلى در زنانى كه تمايل } \\
& \text { به استفاده از داروهاى گياهى دارند به كار برده شود. }
\end{aligned}
$$

\section{References}

1. da Silva PB, dos Santos R, Aparecido M, Bonifácio BV, Negri KMS, Sato MR, et al. Nanotechnological strategies for vaginal administration of drugs-A review. $J$ biomed nanotechnol. 2014;10(9):2218-43.

2. DE S. Genitourinary Infection and Sexually Transmitted Diseases. $15^{\text {th }}$ ed. Philadelphia 2012. p. 560

3. Martins HPR, Da Silva MC, Paiva LCF, Svidzinski TIE, Consolaro MEL. Efficacy of fluconazole and nystatin in the treatment of vaginal Candida species. Acta dermato-venereologica. 2012; 92(1):78-82.

4. Khanjani N, Zamanian M, Molazadeh P, Sadeghi M. The Prevalence of Candida Albicans Infection and Related Factors in Women Referring to Health Centers of Jiroft in 2010: A Short Report. Journal of Rafsanjan University of Medical Sciences. 2014;13(6):569-76. [Persian]

5. Johnson E, Berwald N. Diagnostic utility of physical examination, history, and laboratory evaluation in emergency department patients with vaginal complaints. Ann Emerg Med. 2008;52(3):294-7.

6. Falahati M, Omidi Tabrizib N, Jahaniani F. Anti dermatophyte activities of Eucalyptus camaldulensis in comparison with Griseofulvin. Iranian Journal of Pharmacology and Therapeutics. 2005;4(2):80-3. [Persian]

7. Maha M, Gazia A, Nermeen M. Effect of glabridin on the structure of ileum and pancreas in diabetic rats: a histological, immunohistochemical and ultrastructural study. Nature and Sci. 2012;10(3):78-90.

8. M Messier C, Grenier D. Effect of licorice compounds licochalcone A, glabridin and glycyrrhizic acid on growth and virulence properties of Candida albicans. Mycoses. 2011;54(6):e801-e6.

9. Monavari S, Shamsi Shahrabadi M, Mortazkar P. The study of Antiviral Effects of Glycyrrihza Glabra Extract on HSV. Journal of Medicinal Plants. 2008;4(28):81-6. [Persian]

10. Mahmoudabadi AZ, Iravani M, Khazrei A. Anti-fungal activity of Glycyrrhiza glabra (Licorice) against vaginal isolates of Candida. Biotechnology an Indian Journal. 2009;3(2):75-7. [Persian]

11. Fatima A, Gupta VK, Luqman S, Negi AS, Kumar J, Shanker K, et al. Antifungal activity of Glycyrrhiza glabra extracts and its active constituent glabridin. Phytotherapy Research: An International Journal Devoted to Pharmacological and Toxicological Evaluation of Natural Product Derivatives. 2009;23(8):1190-3.

12. Patel DA, Gillespie B, Sobel JD, Leaman D, Nyirjesy P, Weitz MV, et al. Risk factors for recurrent vulvovaginal candidiasis in women receiving maintenance antifungal therapy: results of a prospective cohort study. Am J Obstet Gynecol. 2004;190(3):644-53. 
13. Rani PU, Naidu MU, Raju GR, Shobha G, Rao TR, Shobha JC, Kumar TV. Multicentric, placebocontrolled, randomised double-blind evaluation of a new herbal cream in Vagial infections. Ancient science of life. 1995 Apr;14(4):212-24.

14. Eslami G, Taheri S, Ayatollahi S, Bagherpour S. Comparison of effects of Glycyrrhiza glabra with nalidixic acid and nitrofurantoin on bacteria isolated from urinary tract infections and urinary stones. Pejouhesh dar Pezeshki. 2010;34(3):178 -81. [Persian]

15. Mohseni R, Omran AN, Norbakhsh F, Rezaie S, Hosseinjani H. A survey of the effect of licorice plant extract on aflR gene expression and aflatoxin production in Aspergillus Parasiticus via Realtime PCR. Pathobiology Research. 2012;15(3):63-77. [Persian]

16. Irani M, Sarmadi M, Bernard F. Leaves antimicrobial activity of Glycyrrhiza glabra L. Iranian journal of pharmaceutical research: IJPR. 2010;9(4):425. [Persian]

17. Sirilun S, Sivamaruthi BS, Kesika P, Pengkumsri N, Tuntisuwanno N, Chaiyasut K, et al. Development and stability evaluation of vaginal suppository containing glycyrrhiza glabra L. For the treatment of Candida albicans infection. DEVELOPMENT. 2018; 11(7).205-9.

18. Arbabi-Kalati F, Porzamani M. Comparison the antifungal effect of licorice and nystatin, invitro study. Journal of Dental Medicine-Tehran University of Medical Sciences. 2013; 26(1):71-4. [Persian] 